\title{
Phyllotaxis-inspired nanosieves with multiplexed orbital angular momentum
}

\author{
Zhongwei Jin ${ }^{1,2 \dagger}$, David Janoschka ${ }^{3 \dagger}$, Junhong Deng ${ }^{4 \dagger}$, Lin Ge ${ }^{5}$, Pascal Dreher ${ }^{3}$, Bettina Frank ${ }^{6}$, Guangwei Hu', \\ Jincheng $\mathrm{Ni}^{1}$, Yuanjie Yang ${ }^{7}$, Jing $\mathrm{Li}^{8}$, Changyuan $\mathrm{Yu}^{1,9}$, Dangyuan Lei ${ }^{10}$, Guixin $\mathrm{Li}^{4}$, Shumin Xiao ${ }^{11}$, \\ Shengtao Mei ${ }^{1}$, Harald Giessen ${ }^{6 *}$, Frank Meyer zu Heringdorf ${ }^{3^{*}}$ and Cheng-Wei Qiu ${ }^{1 *} \mathbb{D}$
}

\begin{abstract}
Nanophotonic platforms such as metasurfaces, achieving arbitrary phase profiles within ultrathin thickness, emerge as miniaturized, ultracompact and kaleidoscopic optical vortex generators. However, it is often required to segment or interleave independent sub-array metasurfaces to multiplex optical vortices in a single nano-device, which in turn affects the device's compactness and channel capacity. Here, inspired by phyllotaxis patterns in pine cones and sunflowers, we theoretically prove and experimentally report that multiple optical vortices can be produced in a single compact phyllotaxis nanosieve, both in free space and on a chip, where one meta-atom may contribute to many vortices simultaneously. The time-resolved dynamics of on-chip interference wavefronts between multiple plasmonic vortices was revealed by ultrafast time-resolved photoemission electron microscopy. Our nature-inspired optical vortex generator would facilitate various vortex-related optical applications, including structured wavefront shaping, free-space and plasmonic vortices, and high-capacity information metaphotonics.
\end{abstract}

Keywords: Phyllotaxis vortex nanosieve, Free-space, Plasmonic vortices, Ultrafast time-resolved

\section{Introduction}

The vortex is ubiquitous in nature, spanning from the galaxy, ocean flow, to phyllotaxis. In electromagnetic wave, the vortex can be found in spiral wavefront profiles, representing the orbital angular momentum (OAM) of light [1-5]. Analogous to the natural vortex, such as tornado and whirlpool, the optical vortices (OVs) carrying OAM have helical phase fronts and

\footnotetext{
*Correspondence: giessen@pi4.uni-stuttgart.de; meyerzh@uni-due.de; chengwei.qiu@nus.edu.sg

${ }^{\dagger}$ Zhongwei Jin, David Janoschka and Junhong Deng authors contributed equally to this work and co-first authors

1 Department of Electrical and Computer Engineering, National University of Singapore, 4 Engineering Drive 3, Singapore 117583, Singapore

${ }^{3}$ Faculty of Physics and Center for Nanointegration Duisburg-

Essen (CENIDE), University of Duisburg-Essen, Lotharstrasse 1-21,

47057 Duisburg, Germany

${ }^{6}$ Physics Institute and Stuttgart Center of Photonics Engineering (SCOPE),

University of Stuttgart, 70569 Stuttgart, Germany

Full list of author information is available at the end of the article
}

donut-shaped intensity profiles [6-10]. The last few decades have witnessed the prosperous development in the advanced application of OVs, such as in quantum optical communications [11], particle manipulation [12-14], super-resolution imaging [15], and multichannel information storage [16-18]. So far, various kinds of optical components have been proposed to generate OAM beams, such as spiral phase plates [19], forked holograms [20], chip-scale microlasers [21-25], diffractive optical components based on conformal logpol mapping $[26,27]$ and multiplane light conversion [28] and ultrathin metasurfaces [29-31]. Recently, multifunctional metasurfaces $[18,29,30,32,33]$ have been investigated to generate multiple OVs within one nanodevice. Such metasurfaces often resort to segmented or interleaved sub-array meta-atoms to multiplex OAMs in a single device. As a result, each inclusion seems only responsible for one specific topological charge. Meanwhile, sufficient distance is required between 
meta-atoms to suppress cross coupling, which in turn degrades the device's compactness and channel capacity. To deal with this problem, one may search therapies/solutions from either frequency domain or space domain. Previously, Elhanan Maguid et al. [29] introduced asymmetric harmonic response geometric phase metasurface which realized OAM multiplexing through superposition of different harmonic components in the momentum space. In this work, we realize both freespace and near-field OAM multiplexing based on structure degeneracy in the space domain.

\section{Results and discussion}

Intriguingly, planar spirals such as Archimedean spirals [34], logarithmic spirals [35] and Fermat spirals [36] can generate photonic OAMs with helical phase fronts. Among the Fermat spirals, the Vogel spiral [37], also known as the "golden ratio" spiral, has been frequently studied for its unique growing pattern [38-40]. The pattern of a Vogel spiral [37] in polar coordinates can be described as $r=c \sqrt{n}, \phi=n \cdot 137.5^{\circ}$. Here, $n$ is the ordering number of a floret (such as the seed in a sunflower pattern), $c$ is a scaling constant, $r$ is the radial distance between the $n^{\text {th }}$ floret and the center of the capitulum (meaning the whole phyllotaxis pattern, such as the whole sunflower pattern), $\phi$ is the angle between the reference direction and the position vector of the $n^{\text {th }}$ floret, and $137.5^{\circ}$ is the "golden angle". The Vogel spiral is well known as one of the phyllotaxis geometries in nature, which exists in many plants including pine cones, sunflower seeds, and so on [38]. As shown in Fig. 1a, multiple sets of clockwise and anti-clockwise spirals can be encoded from such a phyllotaxis geometry pattern. And the numbers of spiral arms contained in different sets are in coincidence with the Fibonacci numbers.

Such interesting phenomenon naturally arouses our interest to investigate the link between the natureinspired pattern and optical vortices. In order to solve this puzzle, we simulated the diffraction pattern of a "golden-ratio" Vogel spiral nanosieve $(c=2.5, n$ starts from 1, ends at 936) in its Fresnel region at $z=300 \mu \mathrm{m}$ upon $633 \mathrm{~nm}$ light's incidence. Indeed, the OAM spectrum analysis [41] reveals that the diffracted pattern of such a mask contains a series of OAM modes (Fig. 1a), in coincidence with the numbers of spiral arms which can be encoded from the pattern. Hence, we infer that phyllotaxis-alike patterns concealing multiple spiral structures may enable the creation and multiplexing of OVs. Such beauty in nature inspires us to design phyllotaxis-alike nanosieves which can generate beams containing multiple OAM modes for both free-space and on-chip optical systems.

\subsection{Design concept of phyllotaxis-inspired nanosieves}

First, we revisit the "vortex comb" phenomenon [42] to obtain the working principle of our phyllotaxis-inspired nanosieves and extend it to both free-space and on-chip optical systems. Under such circumstances, light emitted from each subwavelength nanohole of nanosieves can be approximated as a point source [43,44]. Considering a total of $M(M>1)$ point sources arranged along the azimuthal domain with equal angular separation, light emitted from the $a^{\text {th }}$ nanohole at plane-wave incidence can be decomposed into the summation of a set of orthogonal LG modes [45]:

$$
\begin{aligned}
\phi\left(\rho, \frac{2 \pi \cdot a}{M}, z\right) \propto & \sum_{p=0}^{\infty} \sum_{l=-\infty}^{\infty} c_{p, l} I_{p, l}(\rho, z) \\
& \exp \left[-i l\left(\frac{2 \pi \cdot a}{M}\right)\right] \cdot \exp \left(i \sigma \frac{2 \pi \cdot a}{M}\right)
\end{aligned}
$$

In Eq. (1), the LG modes are written in the form of $I_{p, l}(\rho, z) \exp (i l \theta)$, where $I_{p, l}(\rho, z)$ denotes the complex amplitude of the corresponding LG mode, and $c_{p, l}$ denotes the expanded coefficient. $\rho$ denotes the radial distance of the point source to the original point and $z$ represents the focal distance. In free space, $z>0$, and $\sigma=0$ as long as the incidence is plane wave; while in the on-chip optical system, $z$ can be approximated as zero, and $\sigma= \pm 1$ for right- (RCP) and left-handed circularly polarization (LCP) states, owing to the appropriate spin-to-orbital conversion mechanism. Therefore, the final field distribution can be approximated as the interference of such $M$ point sources, which is given by the summation of these individual elementary waves:

$$
I=\sum_{a=0}^{M-1} \sum_{p=0}^{\infty} \sum_{l=-\infty}^{\infty} c_{p, l} I_{p, l}(\rho, z) \exp \left[-i(l-\sigma) \frac{2 \pi \cdot a}{M}\right]
$$

Since $\sum_{a=0}^{M-1} \exp \left[-i(l-\sigma) \frac{2 \pi \cdot a}{M}\right]$ is the summation of a finite geometric series and can be easily calculated as:

$$
\sum_{a=0}^{M-1} \exp \left[-i(l-\sigma) \frac{2 \pi \cdot a}{M}\right]=\frac{1-\exp \left[-i(l-\sigma) \frac{2 \pi \cdot a M}{M}\right]}{1-\exp \left[-i(l-\sigma) \frac{2 \pi \cdot a}{M}\right]}=\left\{\begin{array}{cc}
M, & l=N M+\sigma \\
0, & l \neq N M+\sigma
\end{array}\right.
$$




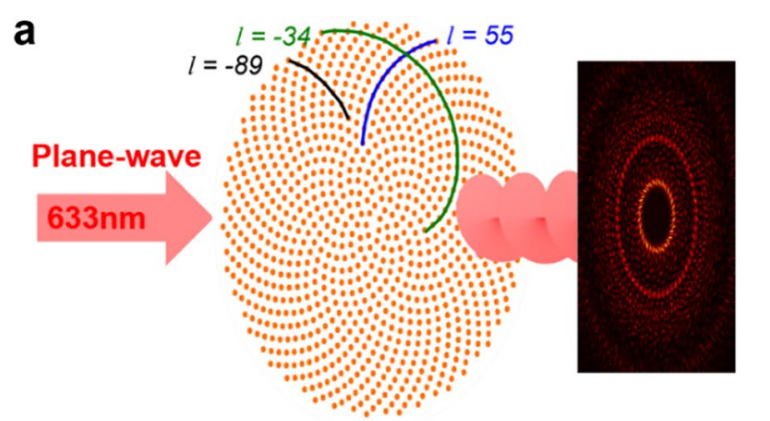

"Golden-ratio" Vogel spiral nanosieve

b

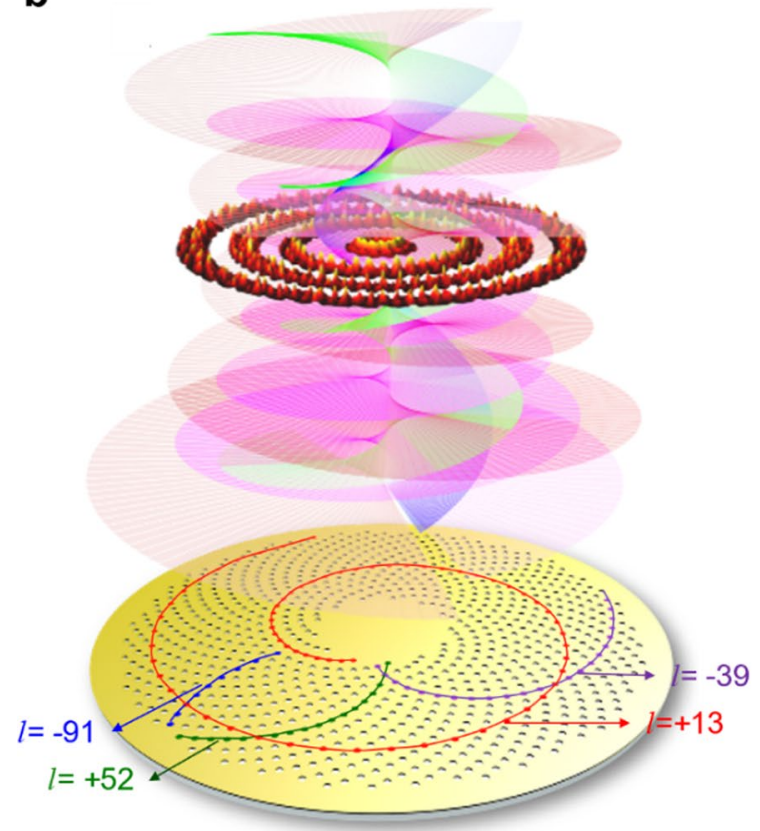

Free-space phyllotaxis vortex nanosieve
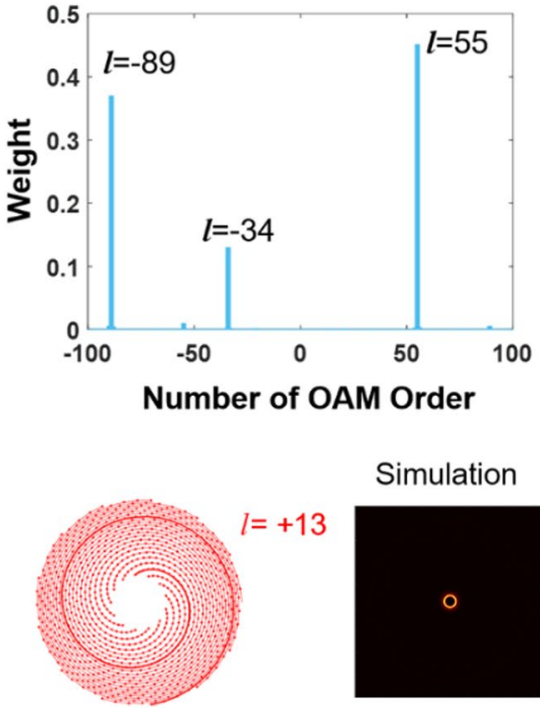

Simulation
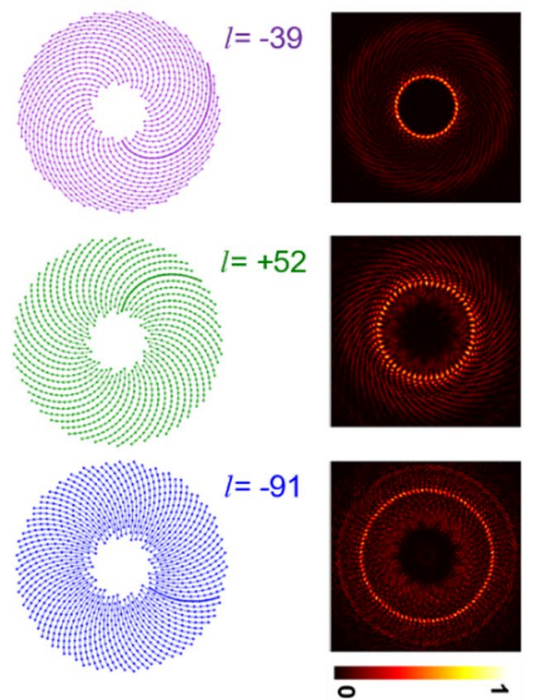

Fig. 1 Free-space phyllotaxis-inspired vortex nanosieve. a A "Golden-ratio" phyllotaxis pattern producing "Fibonacci" OAM series inspires us to design phyllotaxis-alike vortex nanosieves. In the Fresnel region, the diffractive pattern of a Vogel spiral nanosieve with 936 florets is simulated and mode coefficients are calculated. The corresponding results reveal the link between phyllotaxis patterns and optical vortices, thus inspiring us to design phyllotaxis-alike vortex nanosieves. b Free-space phyllotaxis-inspired vortex nanosieve generating four different on-axis optical vortices simultaneously in free-space. Left panel: The red, purple, green, and blue solid circles denote the four different motifs concealed in the free-space phyllotaxis-inspired vortex nanosieve structure. Right panel: The four different sets of concealed spirals and their relevant simulated Fresnel diffraction intensity profiles

where $N$ is an integer, namely $N=0, \pm 1, \pm 2, \pm 3, \ldots$. Combining Eqs. (2) and (3), we can conclude the following: For the free-space optical system, where $\sigma=0$, the interference pattern of such $M$ point sources can be expressed as:

$$
I=\left\{\begin{array}{c}
M \cdot \sum_{l} \sum_{p=0}^{\infty} c_{p, l} I_{p, l}(\rho, z), l=N M \\
0, l \neq N M
\end{array}\right.
$$


while for an on-chip optical system, where $\sigma= \pm 1$, the interference pattern of such $M$ point sources can be expressed as:

$$
I=\left\{\begin{array}{c}
M \cdot \sum_{l} \sum_{p=0}^{\infty} c_{p, l} I_{p, l}(\rho), l=N M+\sigma \\
0, l \neq N M,
\end{array}\right.
$$

where $N=0, \pm 1, \pm 2, \pm 3, \ldots$ in Eqs. (3), (4), and (5).

In brief, we remark two important conclusions from the above theoretical discussion. First, multiple orders of OAM modes can be generated both in free space and in the near field via a single nanosieve device, as visible in Eqs. (4) and (5). The appearance of these sequential OAM orders is deeply rooted in the rearrangement of the nanoholes into different sets of spirals (see various colored spiral lines in Fig. 1a and more details in the following designs), and those spirals will render the corresponding different OAMs. This is also the reason of the emerged Fibonacci sequential OAM orders embedded in a "golden-ratio" phyllotaxis nanosieve, inspired of which we call our compact devices phyllotaxis-inspired vortex nanosieves. Second, in free space, the OAM orders are independent of incident spins; while in the on-chip optical system, we can get a series of OAM modes containing spin-to-orbit conversion. Intrinsically, the surface plasmon polariton (SPP) wave excited via the circular-shape nanohole by circularly polarized light has different initial phases along different propagating directions. However, in the on-chip optical system, only SPP wave propagating towards the center of the nanosieve will interfere and form the vortices. Therefore, under circular polarization illumination, our phyllotaxis-inspired vortex nanosieve realizes spin-to-orbit conversion.

\subsection{Free-space phyllotaxis-inspired vortex nanosieve}

We employed the Fermat spiral with the formulation [36] $r_{\theta}=\sqrt{r_{0}^{2}+2 l z_{0} \lambda \cdot \frac{\theta}{2 \pi}},\left(r_{0} \ll z_{0}\right)$, to generate a beam with tailored OAM modes in the free-space optical system. Here, $\theta$ denotes the azimuthal angle of the spiral, $r_{\theta}$ denotes the spiral radius corresponding to azimuthal angle $\theta$, and $r_{0}$ is the starting radius of the spiral. Therefore, light penetrating the spiral slit structure will form a helical wavefront and accumulate a $l \cdot 2 \pi$ phase difference on the designed focal distance $z_{0}$. Combining our previous derivation along with inspiration from the "golden ratio" phyllotaxis nanosieve, we repeated such spiral structure equally along the azimuthal angular domain $l$ times and segmented the spiral slit structure into azimuthal equally separated nanoholes to obtain a phyllotaxis-inspired vortex nanosieve. Specifically, we choose $\lambda=633 \mathrm{~nm}, r_{0}=22 \mu m, z_{0}=250 \mu m$ and $l=13$.
Here, we vary spiral azimuthal angle covering from 0 to $3 \pi$. Each of the 13 spirals is azimuthal equally segmented into 72 nanoholes. Hence, our phyllotaxis-inspired vortex nanosieve is composed of 936 arranged nanoholes in total (Fig. 1b).

As indicated by our theoretical insight, we now prove that our phyllotaxis-inspired vortex nanosieve can generate multiple OAMs beyond the topological charge of $l$. As the location of each nanohole is fixed, we can re-unite or re-sample the nanohole arrays. If we "string" the neighboring nanoholes following different trajectories, different spiral patterns can be encoded. As is shown in the right panel of Fig. 1b, four obvious sets of motifs can be encoded from the free-space phyllotaxis-inspired vortex nanosieve, which are 13 clockwise spirals, 39 anti-clockwise spirals, 52 clockwise spirals, and 91 anti-clockwise spirals correspondingly. Based on this, we can infer that light coming from our free-space phyllotaxis-inspired vortex nanosieve will simultaneously carry four OAM modes with different helical wavefronts, with the corresponding topological charges of $l=+13,-39,+52$, and - 91, whose numerical amplitude intensity profile is also shown the right panel of Fig. $1 \mathrm{~b}$.

To verify our analysis, both numerical simulation and experiments were carried out. Figure $2 \mathrm{a}, \mathrm{b}$ shows the simulated intensity and phase profiles of the diffraction pattern of the free-space phyllotaxis-inspired vortex nanosieve at $z=250 \mu \mathrm{m}$ upon $633 \mathrm{~nm}$ 's illuminance respectively. It can be clearly observed that the generated on-axis four OAM patterns in Fig. 2a are the superposition of the four simulated modes shown in Fig. 1b with nearly no distortion. Meanwhile, one can directly obtain the modes' information from the corresponding phase profile. The free-space phyllotaxis-inspired vortex nanosieve sample was fabricated using focused-ion beam (FIB) technique on a $120-\mathrm{nm}$ thick Au film above a glass substrate. The radius of each milled nanohole is $1 \mu \mathrm{m}$, so that the maximum phase difference of light coming from the same nanohole to the focal plane can be ignored. The thickness of the Au film is determined considering three factors. One is that the Au film should be thick enough to block light incident on the film where there are no holes. Other reasons are to avoid waveguide phenomenon and to insure fabrication convenience. Figure $2 \mathrm{c}$ shows the top-view scanning electronic microscopic (SEM) picture of the sample. The measured intensity profiles by light beams with different wavelengths (experimental setup can be found in Additional file 1 Part 1: Fig. S1) are shown in Fig. 2d. As mentioned, the designed focal plane is $250 \mu \mathrm{m}$ above the nanosieve for $633 \mathrm{~nm}$ incident light. According to Fresnel's principle, the focal planes would change to $298 \mu \mathrm{m}$ and $356 \mu \mathrm{m}$ for the incident wavelength of $532 \mathrm{~nm}$ and $445 \mathrm{~nm}$, respectively. All the 
a

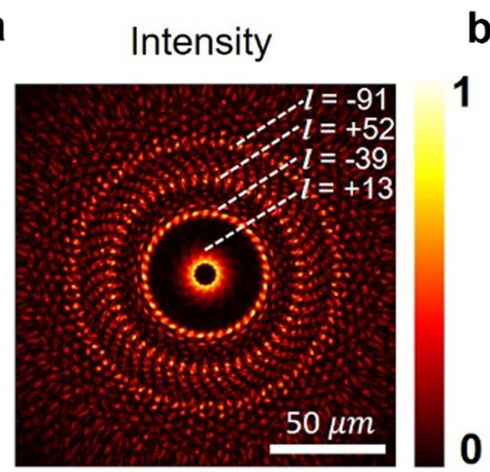

d

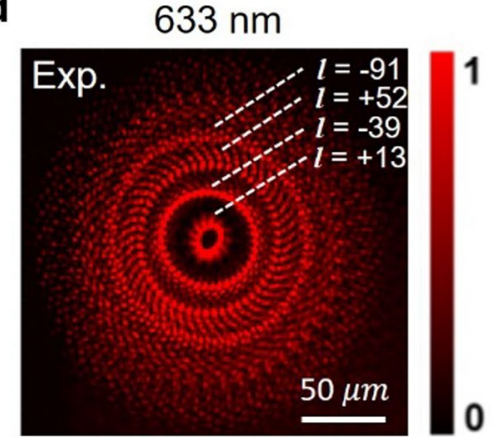

b

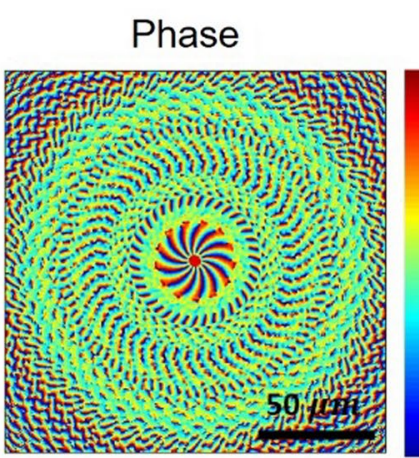

0

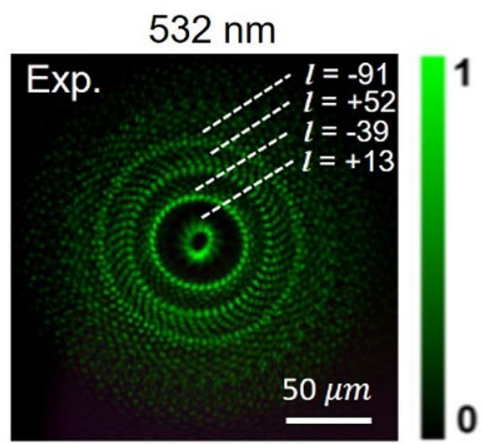

C

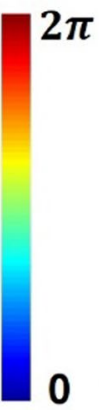

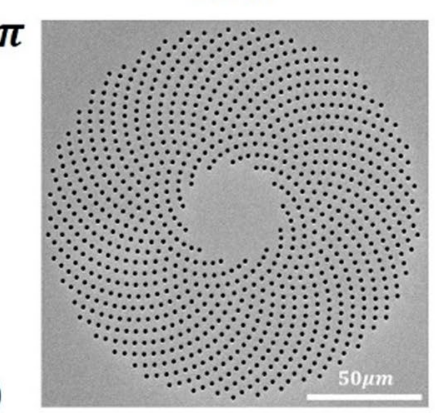

SEM

$445 \mathrm{~nm}$

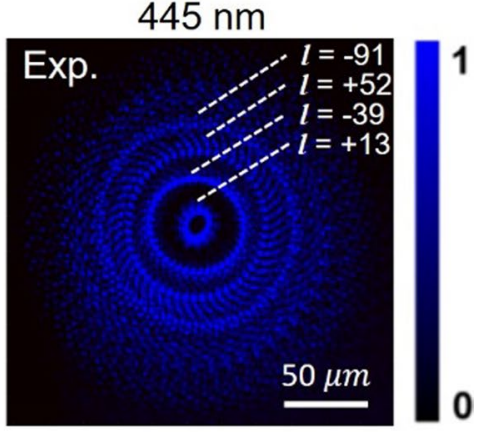

Fig. 2 Simulation and experimental results of the free-space phyllotaxis-inspired vortex nanosieve. a, b Simulated intensity (a) and phase (b) profiles of the free-space phyllotaxis-inspired vortex nanosieve. $\mathbf{c}$ A top-view scanning electron microscope (SEM) image of the sample. $\mathbf{d}$ The measured free-space Fresnel diffraction intensity profiles upon 633 nm, 532 nm, and 445 nm illumination, respectively

intensity profiles are captured near the corresponding focal planes of the nanosieve. It is interesting to know that the OAM mode sequence obtained from our freespace phyllotaxis-inspired vortex nanosieve is actually the first four numbers in the Lucas numbers sequence multiply by 13 , which are 1, 3, 4 and 7 . The Lucas numbers are an integer sequence that are closely related to the more well-known Fibonacci numbers, and are obtained like the Fibonacci series, but with starting values 2 and $1(2,1,3$, $4,7,11, \ldots)$. Statistics showed that $4 \%$ of the patterns of pine trees grown in Norway follow the Lucas numbers, while the majority of the rest follow Fibonacci numbers [46].

\subsection{Plasmonic phyllotaxis-inspired vortex nanosieve}

For the design of the plasmonic phyllotaxis-inspired vortex nanosieve, we employed an Archimedean spiral structure with the formulation $r_{l}(\theta)=r_{0}+\frac{l \theta}{2 \pi} \cdot \lambda_{\text {SPP }}$. Here, $l$ is the designed topological charge; $\theta$ is the azimuthal angle; $r_{0}$ denotes the initial radius of the spiral; $r_{l}(\theta)$ denotes the spiral radius corresponding to azimuthal angle $\theta$ associated with a topological charge of $l$. Besides, $\lambda_{S P P}$ denotes the SPP wavelength which is around $606 \mathrm{~nm}$ (the detail calculation of the SPP wavelength is provided in Additional file 1: Part 6) at the interface of gold and air at the pump laser frequency of $633 \mathrm{~nm}$. In our design, we specifically set $l=40, r_{0}=10 \mu \mathrm{m}$, and $\theta$ from 0 to $\frac{5 \pi}{l}$. To construct the phyllotaxis-inspired vortex nanosieve, 40 such spirals are azimuthal equally arranged, each segmented into 4 nanoholes (Fig. 3a).

As shown in Fig. 3a, such an on-chip phyllotaxisinspired vortex nanosieve could encode 40 anti-clockwise rotated spirals (red solid circles and dotted lines in Fig. 3a) and 80 anti-clockwise rotated spirals (blue solid circles and dotted lines in Fig. 3a). Thus, upon excitation, SPPs excited at each nanohole propagate towards the center, and at least two distinct plasmonic vortex modes would emerge. Considering the spin-orbit conversion [47] suggested by Eq. (5), for right-handed circular polarization (RCP), the generated plasmonic vortex modes should be $-79,-39$, and +1 ; while for the left-handed circular polarization (LCP), the resultant plasmonic vortex modes would be $-81,-41$, and -1 . Note that the vortex modes with $l= \pm 1$ (positive for RCP and negative for LCP incidence) stem from the rearrangement of nanosieve into a circle with the equal radial distance away from the center. Such vortex can also be viewed as a deuterogenic plasmonic vortex [48]. 
In order to obtain accurate optical responses of the plasmonic phyllotaxis-inspired vortex nanosieve, both full-wave numerical simulations based on Lumerical FDTD and near-field measurement using scanning near-field optical microscope (SNOM, Ntegra solaris from NT-MDT Spectrum Instrument, Moscow, Russia) have been carried out. Detailed configurations for both simulation and measurement are provided in Additional file 1 Part 2: Fig. S2. The sample was fabricated using FIB technique through etching $120 \mathrm{~nm}$-thick Au nanoholes on top of glass substrate and the top-view SEM picture of the sample is provided in Fig. 3b. The radius of each nanohole is $150 \mathrm{~nm}$, so that the maximum phase difference of light coming from the same nanohole can be ignored. Figures 3c, d depict numerical intensity and phase profiles under opposite circular polarizations illumination and linear polarization illumination. According to the simulated intensity profiles in Fig. 3c, three on-axis plasmonic vortex modes can be clearly observed, which agrees with our theoretical expectations. Meanwhile, the phase variations shown in Fig. 3d further verify the results. Under RCP illumination, a $2 \pi$ phase change in the center of the phase profile can be clearly observed, which denotes a plasmonic vortex with $l=+1$. Besides, $-39 \cdot 2 \pi$ phase variation and $-79 \cdot 2 \pi$ phase variation can be noticed in the corresponding regions of the phase profile, indicating plasmonic vortices with $l=-39$ and $l=-79$ respectively. Under LCP illumination, $-2 \pi$, $-41 \cdot 2 \pi$ and $-81 \cdot 2 \pi$ phase changes can be observed in the corresponding regions of the phase profile, indicating plasmonic vortices with $l=-1,-41$ and -81 . For linear polarization (LP) incidence, the generated modes are a combination of the modes generated under RCP and LCP incidences as expected. This is because LP can be regarded as linear combination of RCP and LCP and our system is linear. The mode decomposition analysis in Additional file 1 Part 3: Fig. S3 also verifies this. These results are further supported by the experimental results as the measured intensity profiles (Fig. 3e) agree with Fig. 3c, which further justifies the effectiveness of our proposed phyllotaxis-inspired vortex nanosieve.

\subsection{Time-resolved investigation of the plasmonic phyllotaxis-inspired vortex nanosieve}

Note that the results above are the time-averaged field intensity distributions. To further investigate the dynamics of the plasmonic phyllotaxis-inspired vortices, we both simulated (see Additional file 1: Part 5) and measured the dynamic formation process of the plasmonic vortices. We resort to time-resolved two-photon photoemission electron microscopy [49-52] (TR-PEEM) to analyze the spatiotemporal dynamic processes to reveal the fundamentals of our plasmonic phyllotaxisinspired vortex nanosieve. The TR-PEEM process begins with a pump-probe excitation of surface plasmons from nanoholes etched in a single-crystal gold flake, then the interference of the propagating surface plasmon with a probe pulse (Fig. 4a), finally the imaging of the ejected photoelectrons in a photoemission electron microscope (PEEM) [52].

Since the TR-PEEM is combined with a Ti:Sapphire laser system operating at $800 \mathrm{~nm}$ central wavelength, the plasmonic wavelength changes to $\lambda \mathrm{spp} \approx 780 \mathrm{~nm}$ (the detail calculation of the SPP wavelength is provided in Additional file 1: Part 6) and we adjusted our design accordingly. An top-view SEM image of the sample is provided in Fig. 4b. We tested the sample using the TRPEEM system under both RCP and LCP incidences, and the relevant videos are uploaded as Additional file 2, 3. We have summarized the snapshots from the TR-PEEM results under RCP incidence which represent the three main stages of the vortex in dynamics formation, which are formation, revolution, and decay. Figure $4 \mathrm{c}-\mathrm{e}$ are the raw data from the TR-PEEM results. Figure $4 \mathrm{f}-\mathrm{h}$ show processed images that correspond to the delay times used in Fig. 4c-e. Using temporal Fourier filtering at the $1 \omega$ component removes the time independent static backgrounds and leaves us exclusively with the SPP dynamics [53]. The spatiotemporal investigation shows how the excited SPPs propagate both inward and outward along the radial coordinate of the nanosieve. While the outward propagating SPPs finally leave the field of view, the inward propagating SPPs interfere and form the vortices.

\footnotetext{
(See figure on next page.)

Fig. 3 Plasmonic phyllotaxis-inspired vortex nanosieve. a A plasmonic phyllotaxis-inspired vortex nanosieve which can yield three plasmonic vortex modes upon circular polarization states. The stereoscopic 'hot' map of the intensity distribution of the z component of the SPP (surface plasmon polariton) wave is depicted above the sample surface. The red and blue solid circles and dotted lines mark the trajectories of two hidden spiral patterns which can be encoded from the structure. The two sets of hidden spiral patterns can excite plasmonic vortices at I $=40( \pm 1), 80( \pm 1)$ upon circular polarizations. b A top-view SEM image of the sample. The radius of each nanohole is $150 \mathrm{~nm}$. c, d The simulated intensity (c) and phase (d) profiles of generated plasmonic vortex modes excited by RCP, LCP, and LP illumination, respectively. e, Measured intensity profiles of the generated plasmonic vortex modes upon RCP, LCP and LP illumination
} 
a

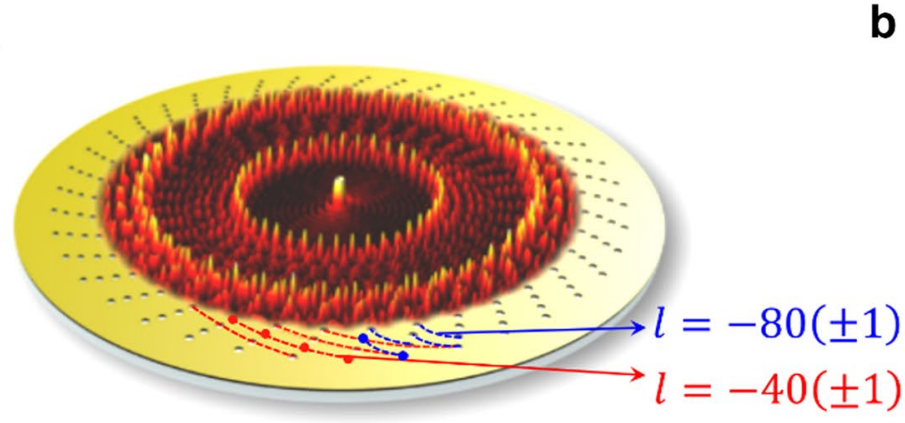

b

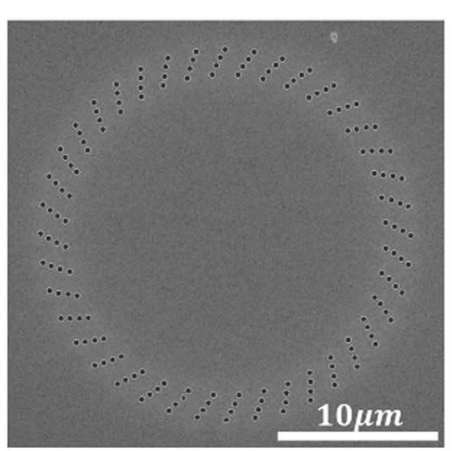

C

RCP

LCP
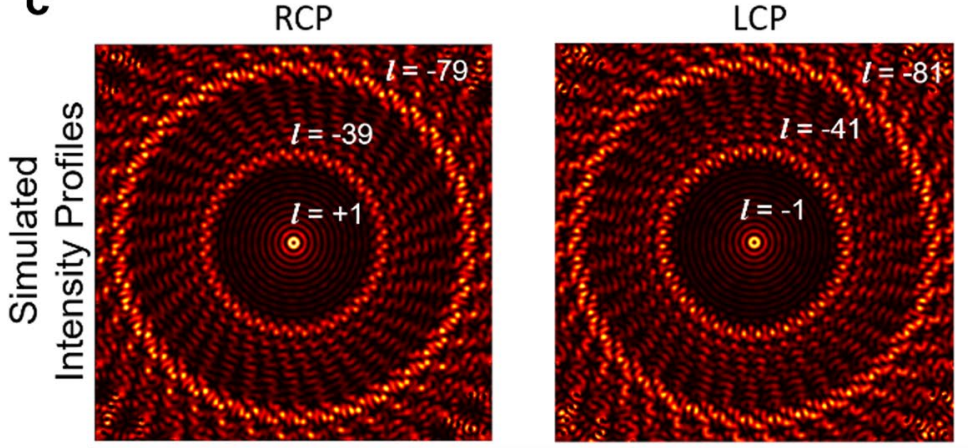

LP
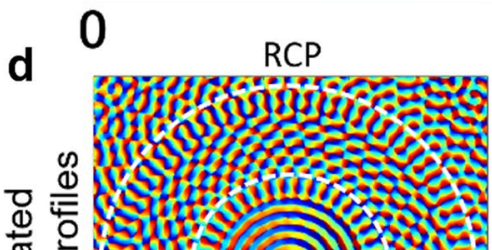

㐫 은

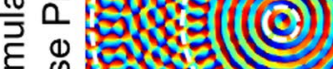

ह ल

ᄃ

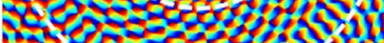
H.

LCP

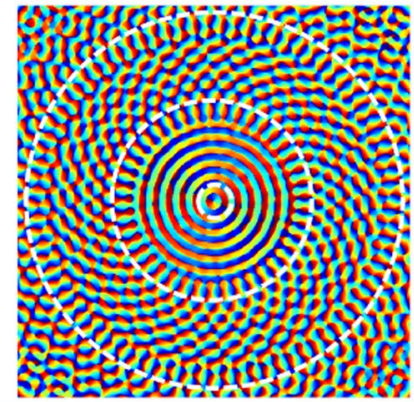

LP

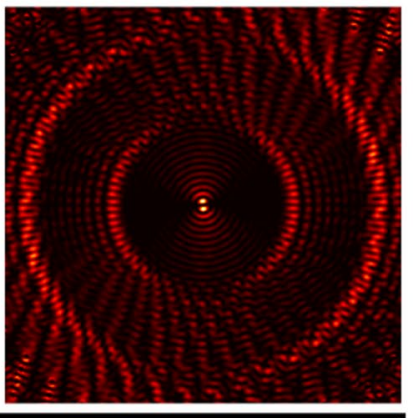

1
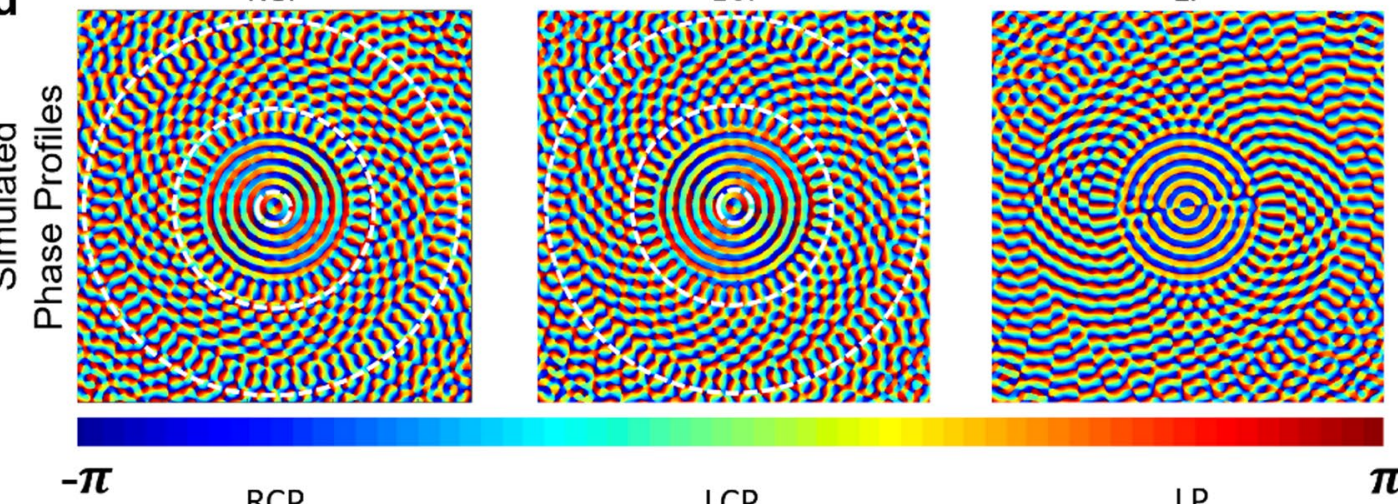

RCP

LCP
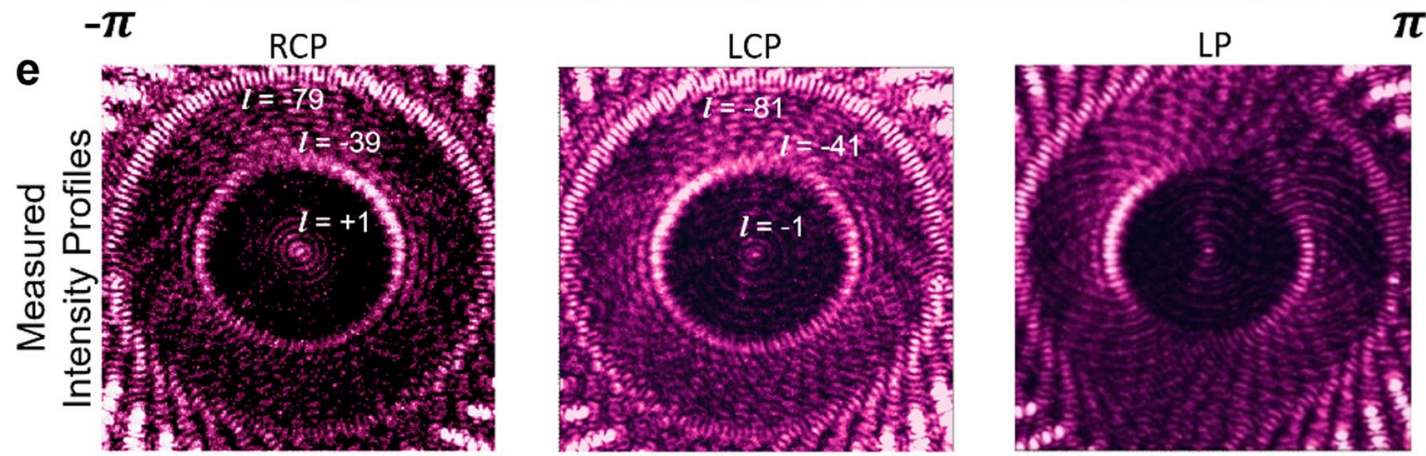

0

Fig. 3 (See legend on previous page.) 


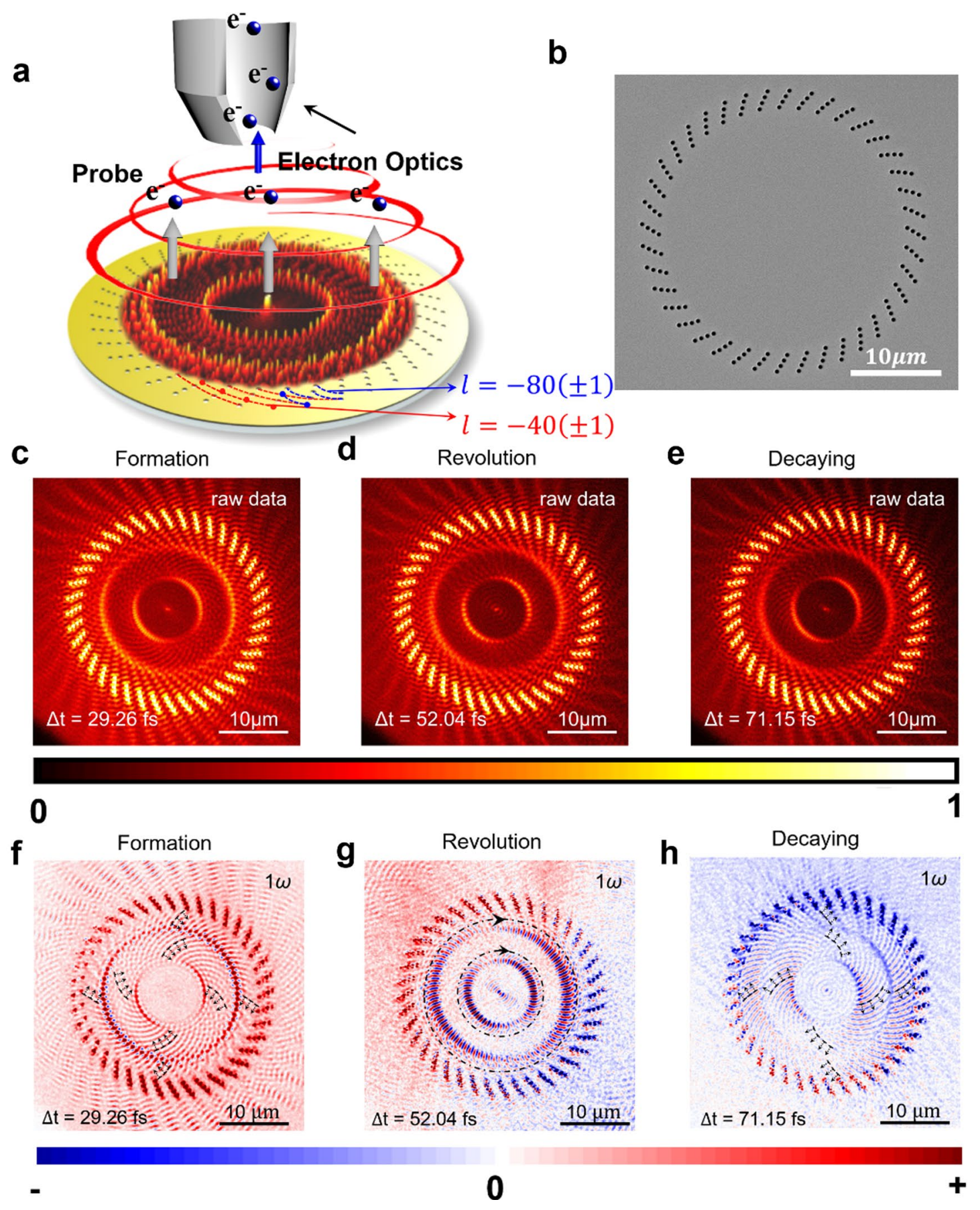

Fig. 4 Dynamic investigation of the plasmonic phyllotaxis-inspired vortex nanosieve. a During the dynamic investigation by TR-PEEM (time-resolved two-photon photoemission electron microscopy), the probe pulse interferes with the propagating SPPs and liberates photoelectrons in a two-photon photoemission process, which will then be imaged with the PEEM setup. b A top-view SEM image of the sample. c-e Raw experimental data of delay-time snapshots from measured TR-PEEM results. Three stages of vortex evolution upon RCP illumination can be identified: formation (c), revolution (d), and decay (e). All the data are shown on a logarithmic intensity scale. $\mathbf{f}, \mathbf{g}$ Processed images with only SPP dynamics that correspond to the delay times used in (c-e). The small black dashed arrows indicate the propagation of vortex wavefronts in each stage. The black dashed arrows in panel $\mathbf{g}$ indicate the revolution direction of the vortex patterns 
According to the handedness of the spiraling wavefronts, we can identify three major stages of vortices' dynamics. In Fig. 4c, $\mathrm{f}$, the converging spiraling wavefronts begin to form the two plasmonic vortices. The handedness of the spiraling wavefronts is indicated by the black dashed arrows in Fig. 4c, f, and agrees with the handedness of the two sets of spirals that comprise our plasmonic phyllotaxis-inspired vortex nanosieve. Subsequently, the resulting revolution of the vortices is depicted in Fig. 4d, $g$, where inward and outward counter-propagating SP waves interfere and form radially standing but azimuthally rotating vortex fields. Finally, the vortices decay and dissolve, forming outward-propagating spiraling wavefronts as displayed in Fig. 4e, h. Compared to the formation stage, the spiraling fringes of the two decaying vortices show inverted handedness.

In the TR-PEEM experiment, the plasmonic vortices are imaged using a pump-probe technique, where both the pump and the probe pulse are circularly polarized. The measured plasmonic vortices in the TR-PEEM experiment thus do not contain the helicity of the pump light [50]. Accordingly, in Fig. 4f-h, the number of lobes of the corresponding plasmonic vortices are 80 and 40 respectively, cancelling the effect of the $\sigma$ term in Eq. 5 . As a result, the plasmonic vortex induced by the pure "spin-orbit conversion" phenomenon is also offset in the measured result. Instead of forming a small "circle" in the center of the measured profiles, a small solid "dot" emerges as the interference result. Additional files 2 and 3 provide the videos of PEEM dynamics of plasmonic phyllotaxis-inspired vortex nanosieve upon LCP and RCP light illumination respectively.

\section{Conclusions}

In conclusion, inspired by the "golden-ratio" spiral phyllotaxis-inspired pattern in nature, we presented the idea of using phyllotaxis-inspired vortex nanosieves to generate optical beams carrying multi-mode vortices both in free space and in a plasmonic near field. Besides theoretical and numerical verifications, we designed and fabricated several phyllotaxis-inspired vortex nanosieves to realize multi-mode vortex manipulation. The phenomenon of multiplexed OAMs in our phyllotaxis-inspired vortex nanosieves comes from the embedded multiple spirals in a single device, each spiral set carrying a different vortex mode, which is confirmed by both steady-state and dynamic-state measured results. Such strategy offers a different recipe with robust multimode OAM manipulation (see Additional file 1: Part 4) for promising application such as on-chip photonic devices [54], optical communication, and even quantum chiral optics.

\section{Methods}

\subsection{Numerical simulation}

The diffractive patterns (intensity and phase profiles) for free-space phyllotaxis-inspired vortex nanosieve and the mode decomposition analysis for both free-space and on-chip phyllotaxis-inspired vortex nanosieve were performed by MATLAB. The simulated static characterization of on-chip phyllotaxis-inspired vortex nanosieve (intensity and phase profiles) was performed by Lumerical FDTD Solution (a commercial software), and the detailed simulation setup is provided in Additional file 1: Part 2.

\subsection{Sample fabrication}

Both the samples used for free-space and static onchip characterization were fabricated on two pieces of 120-nm-thick Au film, respectively. The Au film was deposited on cleaned glass substrate using E-beam evaporation (HHV, AUTO500) with a deposited rate of $1 \AA$ s. Then the pattern for free-space and static onchip characterization was sculptured on $\mathrm{Au}$ film using focused ion beam technique (FEI, Helios NanoLab $600 \mathrm{i})$, which is controlled by the software named NanoBuilder. The current and energy of ion beam are $80 \mathrm{pA}$ and $30 \mathrm{kV}$, respectively. And the dwell time and volume per dose of patterning conditions are $1 \mu \mathrm{s}$ and $0.15 \mu \mathrm{m}^{2} / \mathrm{nC}$ in Nanobuilder, respectively.

For TR-PEEM measurements, we chemically synthesize single crystalline gold platelets [52] on n-doped silicon substrates, which have lateral dimensions of up to one hundred micrometers. We then structure these atomically flat surfaces with phyllotaxis-inspired patterns, using a focused beam of $\mathrm{Au}++$ ions, which is generated by a Raith IonLine plus system.

\subsection{Sample characterization}

The SNOM system used for static on-chip characterization was Ntegra solaris, NT-MDT Spectrum Instrument, Moscow, Russia. The Fiber SNOM probe was NF113NTF, Scansens GmbH, Ostec Instrument, Germany. 
The TR-PEEM system used for investigating the temporal dynamics of the nanosieve was built around a SPE-LEEM III (ELMITEC Elektornenmikroskopie GmbH,Germany) that was combined with a Ti:Sapphire femtosecond laser oscillator (Femtolasers). Pump- and probe pulses were created and mutualy delayed in a home-built Pancharatnam's phase stabilized MachZehnder Interferometer. We work in normal-incidence geometry, as described in Refs $[34,55]$.

\section{Supplementary Information}

The online version contains supplementary material available at https://doi. org/10.1186/s43593-021-00005-9.

Additional file 1: Part 1. Simulation mode decomposition analysis for free-space phyllotaxis-inspired vortex nanosieve and its optical characterization setup. Part 2. Simulation setup and optical setup for static characterization of on-chip phyllotaxis-inspired vortex nanosieve. Part 3. Simulated mode decomposition analysis of the generated plasmonic vortices from the on-chip phyllotaxis-inspired vortex nanosieve. Part 4. The Robustness of the Phyllotaxis-inspired Vortex Nanosieves. Part 5. Simulated dynamic characterization of on-chip phyllotaxis-inspired vortex nanosieve using FDTD. Part 6. Calculation of $\lambda_{\text {spp }}$.

Additional file 2: PEEM dynamics of on-chip nanosieve upon LCP light illumination.

Additional file 3: PEEM dynamics of on-chip nanosieve upon RCP light illumination.

\section{Acknowledgements}

Not applicable.

\section{Authors' contributions}

Z.J. and C.W.Q. conceived the idea. Z.J., C.W.Q., S.M. and S.X. designed the nano-devices and did the optical characterization of the free-space phyllotaxis-inspired vortex nanosieve. J.D. and G.L. did the nanofabrication and took SEM pictures for both free-space phyllotaxis-inspired vortex nanosieve sample and the sample used in the static characterization of the on-chip phyllotaxis-inspired vortex nanosieve. G.L., J.L., D.L. and X.H did the static optical characterization of the on-chip phyllotaxis-inspired vortex nanosieve. D.J and F.M.H. did the dynamic optical characterization of the on-chip phyllotaxisinspired vortex nanosieve. B.F. and H.G. did the nanofabrication of the sample used in dynamic characterization of the phyllotaxis-inspired vortex nanosieve. P.D. did the data analyzation of the dynamic characterization of the on-chip phyllotaxis-inspired vortex nanosieve. G.H., J.N., and C.Y. participated in the discussions and contributed valuable suggestions for the phyllotaxis-inspired vortex nanosieves. The paper was written by Z.J. with inputs from D.J., J.D., L.G., P.D., B.F., H.G., G.H., J.N., and C.W.Q. C.W.Q supervised the project. All authors analyzed the data, read and corrected the paper before paper submission. All authors read and approved the final manuscript. Z.J., D.J. and J.D. contributed equally and should be considered co-first authors. All authors read and approved the final manuscript.

\section{Funding}

This work was supported by the National Research Foundation, Prime Minister's Office, Singapore under Competitive Research Program Award NRFCRP22-2019-0006; the grant (R-261-518-004-720) from Advanced Research and Technology Innovation Centre (ARTIC); the Deutsche Forschungsgemeinschaft (DFG, German Research Foundation) -Project-ID 278162697-SFB 1242; and ERC Advanced Grant Complex Plan, BMBF, DFG and BW-Stiftung; and the Research Grants Council of Hong Kong (CRF Grant No. C6013-18G) and the City University of Hong Kong (Project No. 9610434); and the support from A*STAR under its AME YIRG Grant (Award No. A2084c0172).

\section{Availability of data and materials}

The datasets used and/or analyzed during the current study are available from the corresponding author on reasonable request.

\section{Declarations}

Competing interests

The authors declare that they have no competing interests.

\section{Author details}

${ }^{1}$ Department of Electrical and Computer Engineering, National University of Singapore, 4 Engineering Drive 3, Singapore 117583, Singapore. ${ }^{2} \mathrm{Col}-$ lege of Optical and Electronic Technology, China Jiliang University, Hangzhou 310018, China. ${ }^{3}$ Faculty of Physics and Center for Nanointegration Duisburg-Essen (CENIDE), University of Duisburg-Essen, Lotharstrasse 1-21, 47057 Duisburg, Germany. ${ }^{4}$ Department of Materials Science and Engineering, Shenzhen Institute for Quantum Science and Engineering, Southern University of Science and Technology, Shenzhen 518055, China. ${ }^{5}$ Beijing Qianjunyide Technology Co. Ltd, Beijing 100031, China. ${ }^{6}$ Physics Institute and Stuttgart Center of Photonics Engineering (SCOPE), University of Stuttgart, 70569 Stuttgart, Germany. ${ }^{7}$ School of Physics, University of Electronic, Science and Technology of China, Chengdu 611731, China. ${ }^{8}$ Key Laboratory of Optoelectronic Materials, Technical Institute of Physics and Chemistry, Chinese Academy of Sciences, Beijing 100190, China. ${ }^{9}$ Department of Electronic and Information Engineering, The Hong Kong Polytechnic University, Hung Hom, Kowloon, Hong Kong SAR, China. ${ }^{10}$ Department of Materials Science and Engineering, City University of Hong Kong, 83 Tat Chee Avenue, Kowloon, Hong Kong SAR, China. ${ }^{11}$ State Key Laboratory On Tunable Laser Technology, Ministry of Industry and Information Technology Key Lab of Micro-Nano Optoelectronic Information System, Shenzhen Graduate School, Harbin Institute of Technology, Shenzhen 518055, China.

Received: 28 April 2021 Revised: 27 July 2021 Accepted: 17 August 2021 Published online: 22 September 2021

\section{References}

1. J. Sun, J. Zeng, X. Wang, A.N. Cartwright, N.M. Litchinitser, Concealing with structured light. Sci. Rep. 4, 4093 (2014). https://doi.org/10.1038/srep0 4093

2. L. Du, A. Yang, A.V. Zayats, X. Yuan, Deep-subwavelength features of photonic skyrmions in a confined electromagnetic field with orbital angular momentum. Nat. Phys. 15, 650-654 (2019). https://doi.org/10.1038/ s41567-019-0487-7

3. Y. Shen et al., Optical vortices 30 years on: OAM manipulation from topological charge to multiple singularities. Light. Sci. Appl. (2019). https://doi. org/10.1038/s41377-019-0194-2

4. J. Pan et al., Index-tunable structured-light beams from a laser with an intracavity astigmatic mode converter. Phys. Rev. Appl. 14, 044048 (2020). https://doi.org/10.1103/PhysRevApplied.14.044048

5. F. Lin, X. Qiu, W. Zhang, L. Chen, Seeing infrared optical vortex arrays with a nonlinear spiral phase filter. Opt. Lett. 44, 2298-2301 (2019). https://doi. org/10.1364/OL.44.002298

6. K.Y. Bliokh, F.J. Rodriguez-Fortuno, F. Nori, A.V. Zayats, Spin-orbit interactions of light. Nat. Photon 9, 796-808 (2015). https://doi.org/10.1038/ nphoton.2015.201

7. B. Wang et al., Generating optical vortex beams by momentum-space polarization vortices centred at bound states in the continuum. Nat. Photonics 14, 623-628 (2020). https://doi.org/10.1038/s41566-020-0658-1

8. Y.Tang et al., Harmonic spin-orbit angular momentum cascade in nonlinear optical crystals. Nat. Photonics 14,658-662 (2020). https://doi.org/10. 1038/s41566-020-0691-0

9. K.M. Dorney et al., Controlling the polarization and vortex charge of attosecond high-harmonic beams via simultaneous spin-orbit momentum conservation. Nat. Photonics 13,123-130 (2019). https://doi.org/10.1038/ s41566-018-0304-3 
10. Z. Ji et al., Photocurrent detection of the orbital angular momentum of light. Science 368, 763 (2020). https://doi.org/10.1126/science.aba9192

11. E. Nagali et al., Quantum information transfer from spin to orbital angular momentum of photons. Phys. Rev. Lett. 103, 013601 (2009). https://doi. org/10.1103/PhysRevLett.103.013601

12. S. Mei et al., Evanescent vortex: optical subwavelength spanner. Appl. Phys. Lett. 109, 191107 (2016). https://doi.org/10.1063/1.4967745

13. M. Padgett, R. Bowman, Tweezers with a twist. Nat. Photonics 5, 343-348 (2011). https://doi.org/10.1038/nphoton.2011.81

14. V.G. Shvedov et al., Optical vortex beams for trapping and transport of particles in air. Appl. Phys. A 100, 327-331 (2010). https://doi.org/10. 1007/s00339-010-5860-4

15. Y. Kozawa, D. Matsunaga, S. Sato, Superresolution imaging via superoscillation focusing of a radially polarized beam. Optica 5, 86-92 (2018). https://doi.org/10.1364/OPTICA.5.000086

16. H. Ren et al., Complex-amplitude metasurface-based orbital angular momentum holography in momentum space. Nat. Nanotechnol. 15, 948-955 (2020). https://doi.org/10.1038/s41565-020-0768-4

17. X. Fang, H. Ren, M. Gu, Orbital angular momentum holography for highsecurity encryption. Nat. Photonics 14, 102-108 (2020). https://doi.org/ 10.1038/s41566-019-0560-x

18. H. Ren et al., Metasurface orbital angular momentum holography. Nat. Commun. 10, 2986 (2019). https://doi.org/10.1038/s41467-019-11030-1

19. L. Rego et al., Generation of extreme-ultraviolet beams with time-varying orbital angular momentum. Science 364, eaaw9486 (2019)

20. J.C. Ni et al., Three-dimensional chiral microstructures fabricated by structured optical vortices in isotropic material. Light Sci. Appl 6, e17011 (2017)

21. C. Huang et al., Ultrafast control of vortex microlasers. Science $\mathbf{3 6 7}$, 1018-1021 (2020)

22. H. Sroor et al., High-purity orbital angular momentum states from a visible metasurface laser. Nat. Photonics 14, 498-503 (2020). https://doi.org/ 10.1038/s41566-020-0623-z

23. Z. Zhang et al., Tunable topological charge vortex microlaser. Science 368, 760 (2020). https://doi.org/10.1126/science.aba8996

24. Z. Zhang et al., Ultrafast control of fractional orbital angular momentum of microlaser emissions. Light Sci. Appl. 9, 179 (2020). https://doi.org/10. 1038/s41377-020-00415-3

25. D.Y. Fedyanin, A.V. Krasavin, A.V. Arsenin, A.V. Zayats, Lasing at the nanoscale: coherent emission of surface plasmons by an electrically driven nanolaser. Nanophotonics 9, 3965-3975 (2020). https://doi.org/10. 1515/nanoph-2020-0157

26. W. Li et al., Rapidly tunable orbital angular momentum (OAM) system for higher order Bessel beams integrated in time (HOBBIT). Opt. Express 27, 3920-3934 (2019). https://doi.org/10.1364/OE.27.003920

27. G. Ruffato, M. Massari, G. Parisi, F. Romanato, Test of mode-division multiplexing and demultiplexing in free-space with diffractive transformation optics. Opt. Express 25, 7859-7868 (2017). https://doi.org/10.1364/OE.25. 007859

28. N.K. Fontaine et al., Laguerre-Gaussian mode sorter. Nature. Communications 10, 1865 (2019). https://doi.org/10.1038/s41467-019-09840-4

29. E. Maguid et al., Photonic spin-controlled multifunctional shared-aperture antenna array. Science 352, 1202 (2016). https://doi.org/10.1126/ science.aaf3417

30. E. Maguid et al., Multifunctional interleaved geometric-phase dielectric metasurfaces. Light Sci. Appl. 6, e17027-e17027 (2017). https://doi.org/ $10.1038 /$ ssa.2017.27

31. J. Wang et al., All-dielectric metasurface grating for on-chip multi-channel orbital angular momentum generation and detection. Opt. Express 27, 18794-18802 (2019). https://doi.org/10.1364/OE.27.018794

32. S. Mei et al., Flat helical nanosieves. Adv. Func. Mater. 26, 5255-5262 (2016). https://doi.org/10.1002/adfm.201601345

33. D. Veksler et al., Multiple wavefront shaping by metasurface based on mixed random antenna groups. ACS Photonics 2, 661-667 (2015). https://doi.org/10.1021/acsphotonics.5b00113
34. R.M. Kerber et al., Interaction of an Archimedean spiral structure with orbital angular momentum light. New J. Phys. 20, 095005 (2018). https:// doi.org/10.1088/1367-2630/aae105

35. M.Q. Mehmood et al., Broadband spin-controlled focusing via logarithmic-spiral nanoslits of varying width. Laser Photonics Rev. 9, 674-681 (2015). https://doi.org/10.1002/lpor.201500116

36. Z. Li et al., Generation of high-order optical vortices with asymmetrical pinhole plates under plane wave illumination. Opt. Express 21, 15755-15764 (2013). https://doi.org/10.1364/OE.21.015755

37. H. Vogel, A better way to construct the sunflower head. Math. Biosci. 44, 179-189 (1979). https://doi.org/10.1016/0025-5564(79)90080-4

38. J. Trevino, H. Cao, L. Dal Negro, Circularly symmetric light scattering from nanoplasmonic spirals. Nano Lett. 11, 2008-2016 (2011). https://doi.org/ $10.1021 / \mathrm{nl} 2003736$

39. K. Niu et al., Linear and nonlinear spin-orbital coupling in golden-angle spiral quasicrystals. Opt. Express 28, 334-344 (2020). https://doi.org/10. 1364/OE.373957

40. M. Mihailescu, Natural quasy-periodic binary structure with focusing property in near field diffraction pattern. Opt. Express 18, 12526-12536 (2010). https://doi.org/10.1364/OE.18.012526

41. K. Huang et al., Spiniform phase-encoded metagratings entangling arbitrary rational-order orbital angular momentum. Light Sci. Appl. 7, 17156-17156 (2018). https://doi.org/10.1038/lsa.2017.156

42. Y. Yang, G. Thirunavukkarasu, M. Babiker, J. Yuan, Orbital-angular-momentum mode selection by rotationally symmetric superposition of chiral states with application to electron vortex beams. Phys. Rev. Lett. 119, 094802 (2017). https://doi.org/10.1103/PhysRevLett.119.094802

43. K. Huang et al., Ultrahigh-capacity non-periodic photon sieves operating in visible light. Nat. Commun. 6, 7059 (2015). https://doi.org/10.1038/ ncomms8059

44. S. Mei et al., On-chip discrimination of orbital angular momentum of light with plasmonic nanoslits. Nanoscale 8, 2227-2233 (2016). https://doi.org/ 10.1039/C5NR07374J

45. G. Molina-Terriza, J.P. Torres, L. Torner, Management of the angular momentum of light: preparation of photons in multidimensional vector states of angular momentum. Phys. Rev. Lett. 88, 013601 (2001). https:// doi.org/10.1103/PhysRevLett.88.013601

46. T. Koshy, Fibonacci and Lucas Numbers with Applications (Wiley, Hoboken, 2017), pp. 16-50

47. W.-Y. Tsai et al., Twisted surface plasmons with spin-controlled gold surfaces. Adv. Opt. Mater. 7, 1801060 (2019). https://doi.org/10.1002/adom. 201801060

48. Y. Yang et al., Deuterogenic plasmonic vortices. Nano Lett. 20, 6774-6779 (2020). https://doi.org/10.1021/acs.nanolett.0c02699

49. P. Kahl et al., Normal-incidence photoemission electron microscopy (NIPEEM) for imaging surface plasmon polaritons. Plasmonics 9, 1401-1407 (2014). https://doi.org/10.1007/s11468-014-9756-6

50. G. Spektor et al., Revealing the subfemtosecond dynamics of orbital angular momentum in nanoplasmonic vortices. Science 355, 1187 (2017). https://doi.org/10.1126/science.aaj1699

51. T.J. Davis et al., Subfemtosecond and nanometer plasmon dynamics with photoelectron microscopy: theory and efficient simulations. ACS Photonics 4, 2461-2469 (2017). https://doi.org/10.1021/acsphotonics.7b00676

52. T.J. Davis et al., Ultrafast vector imaging of plasmonic skyrmion dynamics with deep subwavelength resolution. Science 368, eaba6415 (2020). https://doi.org/10.1126/science.aba6415

53. G. Spektor et al., Mixing the light spin with plasmon orbit by nonlinear light-matter interaction in gold. Phys. Rev. X 9, 021031 (2019). https://doi. org/10.1103/PhysRevX.9.021031

54. D. Shaohua et al., On-chip trans-dimensional plasmonic router. Nanophotonics (2020). https://doi.org/10.1515/nanoph-2020-0078

55. P. Kahl et al., Direct observation of surface plasmon polariton propagation and interference by time-resolved imaging in normal-incidence two photon photoemission microscopy. Plasmonics 13, 239-246 (2018) 